\title{
Ärztinnen und Ärzte: unterschiedliche Interessen?
}

\author{
Jürg Schlup \\ Dr. med., Präsident der FMH
}

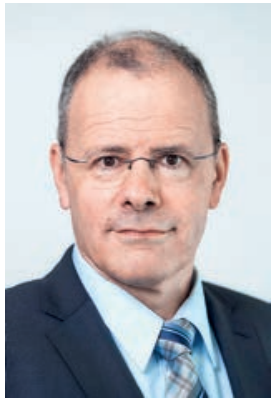

Manche Dinge scheinen auf der Hand zu liegen. So schien es mir bis vor kurzem evident, dass die ungleiche Verteilung der Geschlechter auf die medizinischen Fachdisziplinen in erster Linie unterschiedliche Interessen widerspiegelt. Warum sonst sollten in Bereichen wie der Hausarztmedizin und der Pädiatrie die Kolleginnen proportional oder sogar stark überproportional vertreten sein - in operativen Fächern hingegen unterrepräsentiert?

Ein näherer Blick zeigt jedoch, dass dieses Phänomen vielschichtiger ist. Entscheidungen über die Fachrichtung - wie auch weitere Karriereentscheidungen - werden von vielen Faktoren beeinflusst, wie der Artikel auf Seite 246 aufzeigt: Das Interesse ist ein wichtiger Grund für die Wahl der Fachdisziplin, aber nicht der einzige. Viele von uns haben mehrere Interessen, so

\section{Das Interesse ist ein wichtiger Grund für die} Wahl der Fachdisziplin, aber auch die Rahmenbedingungen können entscheidend sein.

dass auch die Rahmenbedingungen entscheiden, welche wir weiterverfolgen (können) und welche nicht. Dabei dürften vor allem zwei Aspekte bedeutsam sein. Erstens stellen sich Fragen wie: Welche Einschränkungen meines Privatlebens verlangt die Fachrichtung meines Interesses von mir? Und was bin ich bereit und in der Lage zu geben? Und zweitens: Wie kann ich mich in dieser Fachrichtung entwickeln? Werde ich gefördert - oder eher gebremst? Kann ich damit rechnen, in einem fairen Wettstreit um attraktive Positionen berücksichtigt zu werden? Die Antworten auf diese Fragen dürften bei Ärztinnen und Ärzten unterschiedlich ausfallen.

Der erste Aspekt berührt insbesondere die Organisation der Arbeitszeiten. Wer lange Tage und Nächte Patienten operiert und betreut, hat zwangsläufig weniger Zeit für Privates. Wenn diese Zeiten zudem schlecht planbar sind, wird es umso schwieriger, familiäre Pflichten zu übernehmen. Die nachrückende Generation der Ärzteschaft tut gut daran zu hinterfragen, wo diese Arbeitsbedingungen tatsächlich der Notwendigkeit geschuldet sind - und wo lediglich der Tradition und Arbeitskultur: Braucht es wirklich Arbeitswochen von über 50 Stunden, um gut operieren zu lernen? Und warum verbringen Assistenzärzte und -ärztinnen dann so viel Zeit mit Administration? Diese Rahmenbedingungen könnten schon so manchen chirurgisch talentierten Nachwuchs bewogen haben, andere Interessen zu verfolgen.

Familienfreundlichere Arbeitsbedingungen gewinnen nicht nur an Bedeutung, weil seit 2004 eine wachsende Mehrheit der Arztdiplome an Frauen geht. Auch junge Ärzte sind heute anders gefordert: Das Alleinverdienermodell, bei dem Hausfrauen die sehr hohe Arbeitszeit erst ermöglichten, ist heute weitestgehend passé. Mit der Berufstätigkeit von Frauen wird die Vereinbarkeit von Beruf und Familie auch zum Männerthema ganz abgesehen davon, dass allgemein der Zeit für das Privatleben ein höheres Gewicht beigemessen wird. Bleibt der zweite Aspekt der Förderung und Chancen. Hier gereicht es jungen Ärztinnen offenbar vor allem in chirurgischen Disziplinen zum Nachteil, dass man bei ihnen eine Familienpause und eine anschliessende (zeitweise) Reduktion ihres Engagements fürchtet. Dies und auch die noch wenigen weiblichen Vorbilder dürften dazu führen, dass so manche talentierte Chirurgin ihr Interesse nicht weiterverfolgt.

\section{Kann ich damit rechnen, in einem fairen} Wettstreit um attraktive Positionen berücksichtigt zu werden?

Diese Situation verändert sich jedoch. Im Jahr 2017 gingen 49\% der Facharzttitel Chirurgie an Frauen - und auch in klassischen Männerdomänen wie der orthopädischen Chirurgie sind die Kolleginnen auf dem Vormarsch: Obwohl der Frauenanteil in diesem Fach bei nur 9,5\% liegt, wurden im Jahr 2017 31\% dieses Facharzttitels an Orthopädinnen erteilt

Es lohnt also, genauer hinzuschauen und Vorurteile $\mathrm{zu}$ revidieren. Dies ist auch entscheidend, wenn wir neue Lösungen für Weiterbildung und Arbeitszeit suchen, um unseren Nachwuchs nach Leistungsbereitschaft und Talent zu fördern. Bei der Wahl der Fachrichtung sollen dem Interesse keine unnötigen Hürden im Wege stehen! 